\title{
Inflammatory biomarkers at hospital discharge are associated with readmission and death in patients hospitalized for COVID-19
}

\author{
Marleen A. Slim ${ }^{1,2}$ - Brent Appelman ${ }^{1} \cdot$ Marcella C. A. Müller ${ }^{2} \cdot$ Matthijs C. Brouwer $^{3} \cdot$ Alexander P. J. Vlaar $^{2}$. \\ W. Joost Wiersinga ${ }^{1,4}$. Lonneke A. van Vught ${ }^{1,2}$ on behalf of the Amsterdam UMC COVID-19 biobank study group
}

Received: 8 September 2021 / Accepted: 27 September 2021 / Published online: 28 October 2021

(c) The Author(s), under exclusive licence to Springer-Verlag GmbH Germany, part of Springer Nature 2021

\section{Introduction}

Even though the survival of patients admitted with coronavirus disease 2019 (COVID-19) has increased with approximately $20 \%$ over the past year [1], readmission and mortality rates remain high $(19.9 \%$ and $9.1 \%$, respectively, within 2 months after hospital discharge (ward and intensive care unit (ICU)_admissions combined) [2]. In community-acquired pneumonia, elevated interleukin (IL)-6 and IL-10 at hospital discharge are associated with mortality in the subsequent 3 and 6 months, despite initial clinical recovery [3]. We aim to evaluate whether elevated levels of IL-6 and IL-10

Marleen A. Slim

m.a.slim@amsterdamumc.nl

1 Center for Experimental and Molecular Medicine, Amsterdam University Medical Centers, Academic Medical Center, University of Amsterdam, Amsterdam, The Netherlands

2 Department of Intensive Care, Amsterdam University Medical Centers, Academic Medical Center, University of Amsterdam, Amsterdam, The Netherlands

3 Department of Neurology, Amsterdam University Medical Centers, Academic Medical Center, University of Amsterdam, Amsterdam, The Netherlands

4 Department of Internal Medicine, Division of Infectious Diseases, Amsterdam University Medical Centers, Academic Medical Center, University of Amsterdam, Amsterdam, The Netherlands at hospital discharge are associated with readmissions and mortality in the following 12 months in patients with COVID-19.

\section{Methods}

This study was part of the Amsterdam University Medical Centers (UMC) COVID-19 biobank. Patients were prospectively included in the biobank if they were admitted to the Amsterdam UMC with COVID19 and had provided written informed consent or not used the opt-out form. COVID-19 was defined as a positive severe acute respiratory syndrome coronavirus-2 (SARS-CoV-2) polymerase chain reaction (PCR). IL-6 and IL-10 were measured in serial blood samples from March to May 2020 [4]. Patients who died during admission were excluded. Since biomarkers were measured in the first wave in The Netherlands, patients did not receive immunomodulatory therapy. Readmissions and mortality after hospital discharge were ascertained by contacting the general practitioner (GP). Biomarker measurements were done by using a Luminex platform [4]. Normally distributed data were analyzed by a t-test and nonparametric continuous data by Mann-Whitney $U$ test. The ethics committee of the Amsterdam UMC approved the study. 


\section{Results}

One-hundred sixty-one patients who were discharged alive formed our cohort. The mean age was 62 years (SD 11.76), $106(68 \%)$ were male, and patients had an average of one comorbidity (IQR [1-3]). Seventy-five patients (47\%) required ICU care during admission. Thirty-four (21\%) were readmitted (median time to readmission was 29 days, IQR [6-97]), and six (4\%) died (median time to death 85 days, IQR [20-169]) in the 12 months following the initial hospitalization for COVID-19. Twenty-three patients were readmitted once, six patients twice, and five patients three or more times. The primary cause of the first readmission was dyspnea or respiratory insufficiency in fourteen (41\%) patients, cardiovascular disease in seven $(21 \%)$, and other causes in thirteen (38\%) patients. Compared to patients without readmissions and/or mortality after discharge, patients with these adverse outcomes were older $(p=0.031)$ and suffered from more comorbidities $(p=0.001$, Table 1$)$.

At time of hospital discharge, most patients in both groups had zero or one abnormal vital parameter according to Halm's criteria [5] (criteria for clinical stability at hospital discharge). Lymphocytes and platelets were significantly lower at discharge in patients who were readmitted or died in the first 2 months following discharge ( $p=0.002$ and $p=0.007$, respectively). The median concentrations of IL-6 and IL-10 at discharge were significantly higher in patients with these adverse outcomes in the first month $(p=0.005$ and $p<0.001$, respectively) and first 2 months ( $p=0.031$ and $p=0.017$, respectively) following discharge (Fig. 1). At 12 months, the IL- 6 and IL-10 concentration did not show significant differences. Biomarkers representing discharge were measured in the last 4 days before discharge. For the biomarker concentrations, we used 26 age and gendermatched controls from the outpatients clinic, with a mean age of 64 years (SD 15.5) of whom 18 (69\%) were male (Fig. 1).

\section{Discussion}

This study shows that after hospitalization for COVID-19, elevated IL-6 and Il-10 concentrations at time of hospital discharge are associated with increased readmission and/or mortality rates over the subsequent 2 months. A similar association was found for lower lymphocyte and platelet concentration at discharge. Previous studies show that lymphopenia and low platelets have been associated with more severe infection [6] and IL-6 concentration is correlated with COVID-19 severity and in-hospital mortality [7]. Our findings could be of special relevance for patients who did not receive tocilizumab, since this recombinant humanized anti-IL-6 receptor monoclonal antibody inhibits the binding of IL- 6 to both membrane and soluble IL-receptors [8].

This study has several limitations. Biomarkers representing hospital discharge were measured in the 4 days prior to discharge and were available in $70(43 \%)$ patients. Second, we could not ascertain readmissions in ten $(6 \%)$ patients in our cohort. Third, due to the lack of controls without COVID-19, we could not investigate if our findings are also true for other diseases. Fourth, the use of tocilizumab, which has been recommended by the World Health Organization as treatment for severely or critically ill patients with COVID19 [9], will have influence of the IL-6 concentration at discharge. Even so, this study shows that COVID-19 patients with elevated IL-6 and IL-10 levels at hospital discharge were associated with an increased risk of readmission and/ or death up to 2 months after hospital discharge when compared with those with normal circulating biomarkers. 


\section{Appendix}

\section{Collaborators Amsterdam UMC COVID-19 biobank study group}

\begin{tabular}{|c|c|c|c|c|c|c|c|}
\hline Agtmael & Michiel & van & Agtmael & Department of Infectious Diseases & Prof. dr & M.A. van Agtmael & agtmael@amsterdamumc.nl \\
\hline Algera & Anne Geke & & Algera & Department of Intensive Care & Drs & A.G. Algera & a.g.algera@amsterdamumc.nl \\
\hline Appelman & Brent & & Appelman & Department of Infectious Diseases & Drs & B. Appelman & b.appelman@amsterdamumc.nl \\
\hline Baarle & Frank & van & Baarle & Department of Intensive Care & Drs & F.E.H.P. van Baarle & f.e.vanbaarle@amsterdamumc.nl \\
\hline Bax & Diane & & Bax & Experimental Immunology & Drs & D.J.C. Bax & d.j.bax@amsterdamumc.nl \\
\hline Beudel & Martijn & & Beudel & Department of Neurology & $\operatorname{Dr}$ & M. Beudel & m.beudel@amsterdamumc.nl \\
\hline Bogaard & Harm Jan & & Bogaard & Department of Pulmonology & Prof. dr & H J Bogaard & hj.bogaard@amsterdamumc.nl \\
\hline Bomers & Marije & & Bomers & Department of Infectious Diseases & $\operatorname{Dr}$ & M. Bomers & m.bomers@amsterdamumc.nl \\
\hline Bonta & Peter & & Bonta & Department of Pulmonology & Dr & P.I. Bonta & p.i.bonta@amsterdamumc.nl \\
\hline Bos & Lieuwe & & Bos & Department of Intensive Care & $\operatorname{Dr}$ & L.D.J. Bos & 1.d.bos@amsterdamumc.nl \\
\hline Botta & Michela & & Botta & Department of Intensive Care & Drs & M. Botta & m.botta@amsterdamumc.nl \\
\hline Brabander & Justin & de & Brabander & Department of Infectious Diseases & Drs & J. de Brabander & j.debrabander@amsterdamumc.nl \\
\hline Bree & Godelieve & & Bree & Department of Infectious Diseases & $\operatorname{Dr}$ & G.J. de Bree & g.j.debree@amsterdamumc.nl \\
\hline Bruin & Sanne & de & Bruin & Department of Intensive Care & Drs & S. de Bruin & s.debruin1@amsterdamumc.nl \\
\hline Bugiani & Marianna & & Bugiani & Department of Pathology & $\operatorname{Dr}$ & M. Bugiani & m.bugiani@amsterdamumc.nl \\
\hline Bulle & Esther & & Bulle & Department of Intensive Care & Drs & E.B. Bulle & e.b.bulle@amsterdamumc.nl>. \\
\hline Chouchane & Osoul & & Chouchane & Department of Infectious Diseases & Drs & O. Chouchane & o.chouchane@amsterdamumc.nl \\
\hline Cloherty & Alex & & Cloherty & Experimental Immunology & Drs & A.P.M. Cloherty & a.p.cloherty@amsterdamumc.nl \\
\hline David & Buis T.P & & Buis & Department of Infectious Diseases & Drs & D.Buis & d.t.p.buis@amsterdamumumc.nl \\
\hline de Rotte & $\begin{array}{l}\text { Maurits } \\
\text { C.F.J }\end{array}$ & & de Rotte & Department of Clinical Chemistry & $\mathrm{dr}$ & M. C.F.J. de Rotte & m.derotte@amsterdamumc.nl \\
\hline Dijkstra & Mirjam & & Dijkstra & Department of Clinical Chemistry & & M. Dijkstra & mirjam.dijkstra@amsterdamumc.nl \\
\hline Dongelmans & Dave A & & Dongelmans & Department of Intensive Care & $\operatorname{Dr}$ & D.A. Dongelmans & d.a.dongelmans@amsterdamumc.nl \\
\hline Dujardin & RomeinW.G & & Dujardin & Department of Intensive Care & & R.W.G Dujardin & r.w.dujardin@amsterdamumc.nl \\
\hline Elbers & Paul & & Elbers & Department of Intensive Care & $\operatorname{Dr}$ & P.E. Elbers & p.elbers@amsterdamumc.nl \\
\hline Fleuren & Lucas & & Fleuren & Department of Intensive Care & Drs & L.M. Fleuren & 1.fleuren@amsterdamumc.nl \\
\hline Geerlings & Suzanne & & Geerlings & Department of Infectious Diseases & Prof. dr & S.E. Geerlings & s.e.geerlings@amsterdamumc.nl \\
\hline Geijtenbeek & Theo & & Geijtenbeek & $\begin{array}{l}\text { Department of Experimental } \\
\text { Immunology }\end{array}$ & $\underline{\text { Prof. dr }}$ & T.B.H. Geijtenbeek & t.b.geijtenbeek@amsterdamumc.nl \\
\hline Girbes & Armand & & Girbes & Department of intensive care & Prof. dr & A.R.J. Girbes & arj.girbes@amsterdamumc.nl \\
\hline Goorhuis & Bram & & Goorhuis & Department of Infectious Diseases & Dr & A. Goorhuis & a.goorhuis@amsterdamumc.nl \\
\hline Grobusch & Martin P & & Grobusch & Department of Infectious Diseases & Prof. dr & M.P. Grobusch & m.p.grobusch@amsterdamumc.nl \\
\hline Hafkamp & Florianne & & Hafkamp & $\begin{array}{l}\text { Department of Experimental } \\
\text { Immunology }\end{array}$ & Drs & F.M.J. Hafkamp & f.m.hafkamp@amsterdamumc.nl \\
\hline Hagens & Laura & & Hagens & Department of Intensive Care & Drs & L.A. Hagens & 1.a.hagens@amsterdamumc.nl \\
\hline Hamann & Jorg & & Hamann & $\frac{\text { Amsterdam UMC Biobank Core }}{\text { Facility }}$ & Dr & J. Hamann & j.hamann@amsterdamumc.nl \\
\hline Harris & Vanessa & & Harris & Department of Infectious Diseases & $\operatorname{Dr}$ & V. C. Harris & v.c.harris@amsterdamumc.nl \\
\hline Hemke & Robert & & Hemke & Department of Radiology & $\operatorname{Dr}$ & R. Hemke & r.hemke@amsterdamumc.nl \\
\hline Hermans & Sabine M & & Hermans & Department of Infectious Diseases & $\operatorname{Dr}$ & S.M. Hermans & s.m.hermans@amsterdamumc.nl \\
\hline Heunks & Leo & & Heunks & Department of Intensive Care & Dr & L.M.A. Heunks & 1.heunks@amsterdamumc.nl \\
\hline Hollmann & Markus & & Hollmann & Department of Anesthesiology & Prof. dr & m.w.Hollmann & m.w.hollmann@amsterdamumc.nl \\
\hline Horn & Janneke & & Horn & Department of Intensive Care & $\operatorname{Dr}$ & J. Horn & j.horn@amsterdamumc.nl \\
\hline Hovius & Joppe W & & Hovius & Department of Infectious Diseases & Prof. dr & J.W. Hovius & j.w.hovius@amsterdamumc.nl \\
\hline Jong & Menno D & de & Jong & Department of Medical Microbiology & Prof. dr & M.D. de Jong & m.d.dejong@amsterdamumc.nl \\
\hline Koning & Rutger & & Koning & Department of Neurology & Drs & R. Koing & r.koning1@amsterdamumc.nl \\
\hline Lim & Endry H.T & & Lim & Department of Intensive Care & Drs & E.H.T. Lim & e.lim@amsterdamumc.nl \\
\hline Mourik & Niels & van & Mourik & Department of Intensive Care & Drs & N. van Mourik & n.vanmourik@amsterdamumc.nl \\
\hline
\end{tabular}




\begin{tabular}{|c|c|c|c|c|c|c|c|}
\hline Agtmael & Michiel & van & Agtmael & Department of Infectious Diseases & Prof. dr & M.A. van Agtmael & agtmael@amsterdamumc.nl \\
\hline Nellen & Jeannine & & Nellen & Department of Infectious Diseases & $\mathrm{Dr}$ & J.F Nellen & f.j.nellen@amc.uva.nl \\
\hline Nossent & Esther J & & Nossent & Department of Pulmonology & Dr & E.J. Nossent & e.nossent@amsterdamumc.nl \\
\hline Paulus & Frederique & & Paulus & Department of Intensive Care & Dr & F. Paulus & f.paulus@amsterdamumc.nl \\
\hline Peters & Edgar & & Peters & Department of Infectious Diseases & peter & E. Peters & e.peters@amsterdamumc.nl \\
\hline Piña-Fuentes & Dan A.I & & Piña-Fuentes & Department of neurology & Drs & D.Piña-Fuentes & d.a.i.pinafuentes@amsterdamumc.nl \\
\hline Poll & Tom & van der & Poll & Department of Infectious Diseases & $\underline{\text { Prof. dr }}$ & T. van der Poll & t.vanderpoll@amsterdamumc.nl \\
\hline Preckel & Bennedikt & & Preckel & Department of Anesthesiology & Prof. dr & b.preckel & b.preckel@amsterdamumc.nl \\
\hline Prins & Jan M & & Prins & Department of Infectious Diseases & Prof. dr & J.M. Prins & j.m.prins@amc.uva.nl \\
\hline Raasveld & Jorinde & & Raasveld & Department of Intensive Care & Drs & s.j.raasveld & s.j.raasveld@amsterdamumc.nl \\
\hline Reijnders & Tom & & Reijnders & Department of Infectious Diseases & Drs & T.D.Y. Reijnders & $\underline{\text { t.d.reijnders@amsterdamumc.nl }}$ \\
\hline Schinkel & Michiel & & Schinkel & Department of Infectious Diseases & Drs & M. Schinkel & m.schinkel@amsterdamumc.nl \\
\hline Schrauwen & Femke A.P & & Schrauwen & Department of Clinical Chemistry & & F.A.P. Schrauwen & f.a.schrauwen@amsterdamumc.nl \\
\hline Schultz & Marcus J & & Schultz & Department of Intensive Care & $\underline{\text { Prof. dr }}$ & M.J. Schultz & m.j.schultz@amsterdamumc.nl \\
\hline Schuurman & Alex & & Schuurman & Department of Internal Medicine & Drs & A.R. Schuurman & a.r.schuurman@amsterdamumc.nl \\
\hline Schuurmans & Jaap & & Schuurmans & Department of Intensive Care & Drs & J. Schuurmans & j.schuurmans2@amsterdamumc.nl \\
\hline Sigaloff & Kim & & Sigaloff & Department of Infectious Diseases & Dr & K. Sigaloff & k.sigaloff@amsterdamumc.nl \\
\hline Slim & Marleen A & & Slim & $\begin{array}{l}\text { Department of Intensive Care and } \\
\text { Infectious Diseases }\end{array}$ & Drs & M.A. Slim & m.a.slim@amsterdamumc.nl \\
\hline Smeele & Patrick & & Smeele & Department of Pulmonology & Drs & P. Smeele & p.smeele@amsterdamumc.nl \\
\hline Smit & Marry & & Smit & Department of Intensive Care & Drs & M.R. Smit & m.r.smit@amsterdamumc.nl \\
\hline Stijnis & Cornelis S & & Stijnis & Department of Infectious Diseases & Dr & C. Stijnis & c.stijnis@amsterdamumc.nl \\
\hline Stilma & Willemke & & Stilma & Department of Intensive Care & Drs & W. Stilma & w.stilma@hva.nl \\
\hline Teunissen & Charlotte & & Teunissen & Neurochemical Laboratory & Prof. dr & C.E. Teunissen & c.teunissen@amsterdamumc.nl \\
\hline Thoral & Patrick & & Thoral & Department of Intensive Care & Drs & P. Thoral & p.thoral@amsterdamumc.nl \\
\hline Tsonas & Anissa M & & Tsonas & Department of Intensive Care & Drs & A.M. Tsonas & a.m.tsonas@amsterdamumc.nl \\
\hline Tuinman & Pieter R & & Tuinman & Department of Intensive Care & Dr & P.R. Tuinman & p.tuinman@amsterdamumc.nl \\
\hline Valk & Marc & van der & Valk & Department of Infectious Diseases & Dr & M. van der Valk & m.vandervalk@amsterdamumc.nl \\
\hline Veelo & Denise & & Veelo & Department of Anesthesiology & Dr & d.p.veelo & d.p.veelo@amsterdamumc.nl \\
\hline Volleman & Carolien & & Volleman & Department of Intensive Care & & C. Volleman & c.volleman@amsterdamumc.nl \\
\hline Vries & Heder & de & Vries & Department of Intensive Care & Drs & H. de Vries & h.vries@amsterdamumc.nl \\
\hline Vught & Lonneke A & & Vught & $\begin{array}{l}\text { Department of Intensive Care and } \\
\text { Infectious Diseases }\end{array}$ & Dr & L.A. van Vught & 1.a.vanvught@amsterdamumc.nl \\
\hline$\overline{\text { Vugt }}$ & Michèle & van & Vugt & Department of Infectious Diseases & Prof. dr & M. van Vugt & m.vanvugt@amsterdamumc.nl \\
\hline Wouters & Dorien & & Wouters & Department of Clinical Chemistry & & D. Wouters & d.wouters@amsterdamumc.nl \\
\hline Zwinderman & A. $\mathrm{H}(\mathrm{Koos})$ & & Zwinderman & $\begin{array}{l}\text { Department of Clinical Epidemiol- } \\
\text { ogy, Biostatistics and Bioinfor- } \\
\text { matics }\end{array}$ & Prof. dr & A.H. Zwinderman & a.h.zwinderman@amsterdamumc.nl \\
\hline Brouwer & Matthijs C & & Brouwer & Department of Neurology & Dr & M.C. Brouwer & m.c.brouwer@amsterdamumc.nl \\
\hline Wiersinga & W. Joost & & Wiersinga & Department of Infectious Diseases & Prof. dr & W.J. Wiersinga & w.j.wiersinga@amsterdamumc.nl \\
\hline Vlaar & $\begin{array}{l}\text { Alexander } \\
\text { P.J }\end{array}$ & & Vlaar & Deparment of Intensive Care & Dr & A.P.J. Vlaar & a.p.vlaar@amsterdamumc.nl \\
\hline Beek & Diederik & van de & Beek & Department of Neurology & Prof. dr & D. van de Beek & d.vandebeek@amsterdamumc.nl \\
\hline
\end{tabular}


Table 1 Clinical characteristics, stratified for readmissions and/or mortality in the first 2 months and 12 months after discharge

\begin{tabular}{|c|c|c|c|c|c|c|}
\hline & \multicolumn{3}{|l|}{ Short term (2 months) } & \multicolumn{3}{|l|}{ Long term (12 months) } \\
\hline & $\begin{array}{l}\text { Readmission and/or } \\
\text { mortality }(n=23)\end{array}$ & $\begin{array}{l}\text { No readmission and/or } \\
\text { mortality }(n=138)\end{array}$ & $P$ value & $\begin{array}{l}\text { Readmission and mor- } \\
\text { tality }(n=37)\end{array}$ & $\begin{array}{l}\text { No readmission and/ } \\
\text { or mortality }(n=124)\end{array}$ & $P$ value \\
\hline \multicolumn{7}{|l|}{ Demographics } \\
\hline Age, mean (SD) & $68.07(12.67)$ & $60.96(11.33)$ & 0.007 & $65.62(13.33)$ & $60.88(11.08)$ & $\mathbf{0 . 0 3 1}$ \\
\hline $\begin{array}{l}\text { Gender, male, no. } \\
(\%)\end{array}$ & $16(69.6 \%)$ & $90(65.2 \%)$ & 0.865 & $24(64.9 \%)$ & $82(66.1 \%)$ & 1.000 \\
\hline BMI, median [IQR] & $27.46[24.56,29.23]$ & $27.75[25.22,32.14]$ & 0.161 & $27.71[24.57,30.97]$ & $27.36[25.19,31.87]$ & 0.690 \\
\hline $\begin{array}{l}\text { Number of } \\
\text { comorbidities }^{1} \text {, } \\
\text { median [IQR] }\end{array}$ & $3.00[1.50,4.00]$ & $1.00[0.00,3.00]$ & 0.001 & $3.00[1.00,4.00]$ & $1.00[0.00,3.00]$ & 0.001 \\
\hline \multicolumn{7}{|l|}{ Admission } \\
\hline $\begin{array}{l}\text { qSOFA, median } \\
\text { [IQR] }\end{array}$ & $1.00[0.00,1.00]$ & $1.00[0.50,1.00]$ & 0.051 & $1.00[0.00,1.00]$ & $1.00[0.00,1.00]$ & 0.648 \\
\hline $\begin{array}{l}\text { MEWS, median } \\
{[\text { IQR] }}\end{array}$ & $2.00[1.00,4.00]$ & $4.00[2.00,5.00]$ & $\mathbf{0 . 0 3 3}$ & $3.00[1.00,5.00]$ & $4.00[2.00,5.00]$ & 0.302 \\
\hline $\begin{array}{l}\text { CT Severity Score }{ }^{2}, \\
\text { mean (SD) }\end{array}$ & $10.59(6.62)$ & $12.73(5.64)$ & 0.177 & $11.57(7.26)$ & $12.58(5.34)$ & 0.476 \\
\hline $\begin{array}{l}\text { Days between onset } \\
\text { and admission, } \\
\text { median [IQR] }\end{array}$ & $10.00[7.75,14.00]$ & $10.00[7.00,14.00]$ & 0.828 & $10.00[7.00,14.00]$ & $10.00[7.00,14.00]$ & 0.860 \\
\hline $\begin{array}{l}\text { Do not resuscitate } \\
\text { order at admission }{ }^{3} \text {, } \\
\text { no. }(\%)\end{array}$ & $15(71.4 \%)$ & $14(14.7 \%)$ & $<0.001$ & $18(60.0 \%)$ & $11(12.8 \%)$ & $<0.001$ \\
\hline $\begin{array}{l}\text { Do not intubate order at } \\
\text { admission }^{3} \text {, no. }(\%)\end{array}$ & $9(42.9 \%)$ & $8(8.4 \%)$ & $<0.001$ & $11(36.7 \%)$ & $6(7.0 \%)$ & $<0.001$ \\
\hline \multicolumn{7}{|l|}{ Discharge } \\
\hline $\begin{array}{l}\text { Length of hospital } \\
\text { stay (days), median } \\
\text { [IQR] }\end{array}$ & $6.00[4.00,8.00]$ & $11.00[6.00,22.00]$ & 0.002 & $7.00[5.00,17.00]$ & $11.00[6.00,20.00]$ & 0.121 \\
\hline $\begin{array}{l}\text { Discharge location, } \\
\text { no. }(\%)\end{array}$ & & & 0.003 & & & 0.047 \\
\hline Home & $11(47.8 \%)$ & $56(40.6 \%)$ & & $17(45.9 \%)$ & $50(40.3 \%)$ & \\
\hline Nursing home & $3(13.0 \%)$ & $1(0.7 \%)$ & & $3(8.1 \%)$ & $1(0.8 \%)$ & \\
\hline Other & $2(8.7 \%)$ & $6(4.3 \%)$ & & $3(8.1 \%)$ & $5(4.0 \%)$ & \\
\hline Rehabilitation & $5(21.7 \%)$ & $66(47.8 \%)$ & & $11(29.7 \%)$ & $60(48.4 \%)$ & \\
\hline $\begin{array}{l}\text { Abnormal Halm's } \\
\text { criteria for } \\
\text { clinical stability at } \\
\text { discharge }^{4,6} \text {, no. }(\%)\end{array}$ & & & 0.499 & & & 0.462 \\
\hline 0 & $10(55.6)$ & $57(60.0)$ & & $15(60.0)$ & $52(59.1)$ & \\
\hline 1 & $8(44.4)$ & $33(34.7)$ & & $10(40.0)$ & $31(35.2)$ & \\
\hline 2 & $0(0.0)$ & $5(5.3)$ & & $0(0.0)$ & $5(5.7)$ & \\
\hline \multicolumn{7}{|c|}{ Complications during admission } \\
\hline $\begin{array}{l}\text { Venous thromboem- } \\
\text { bolism, no. }(\%)\end{array}$ & $6(26.1)$ & $38(27.5)$ & 1.000 & $10(27.0)$ & $34(27.4)$ & 1.000 \\
\hline $\begin{array}{l}\text { Required ICU stay, } \\
\text { no. }(\%)\end{array}$ & $5(21.7)$ & $70(50.7)$ & 0.019 & $13(35.1)$ & $62(50.0)$ & 0.161 \\
\hline $\begin{array}{l}\text { Mechanical ventila- } \\
\text { tion, no. }(\%)\end{array}$ & $4(17.4)$ & $67(48.9)$ & 0.010 & $12(33.3)$ & $59(47.6)$ & 0.185 \\
\hline \multicolumn{7}{|c|}{ Laboratory values at discharge $^{5}$} \\
\hline $\begin{array}{l}\text { White blood cell } \\
\text { count }\left(10^{\wedge} 9 / \mathrm{L}\right), \\
\text { median }(\mathrm{SD})\end{array}$ & $6.14(2.34)$ & $6.89(2.34)$ & 0.497 & $6.57(2.54)$ & $6.88(2.31)$ & 0.707 \\
\hline
\end{tabular}


Table 1 (continued)

\begin{tabular}{|c|c|c|c|c|c|c|}
\hline & \multicolumn{3}{|l|}{ Short term (2 months) } & \multicolumn{3}{|l|}{ Long term (12 months) } \\
\hline & $\begin{array}{l}\text { Readmission and/or } \\
\text { mortality }(n=23)\end{array}$ & $\begin{array}{l}\text { No readmission and/or } \\
\text { mortality }(n=138)\end{array}$ & $P$ value & $\begin{array}{l}\text { Readmission and mor- } \\
\text { tality }(n=37)\end{array}$ & $\begin{array}{l}\text { No readmission and/ } \\
\text { or mortality }(n=124)\end{array}$ & $P$ value \\
\hline $\begin{array}{l}\text { Lymphocytes } \\
\left(10^{\wedge} 9 / \mathrm{L}\right), \text { median } \\
{[\mathrm{IQR}]}\end{array}$ & $0.68[0.61,0.70]$ & $1.33[1.07,1.94]$ & 0.007 & $0.70[0.66,1.45]$ & $1.33[1.07,1.94]$ & 0.103 \\
\hline $\begin{array}{l}\text { Neutrophils } \\
\left(10^{\wedge} 9 / \mathrm{L}\right), \text { median } \\
{[\mathrm{IQR}]}\end{array}$ & $3.96[3.18,4.99]$ & $4.35[3.00,5.36]$ & 0.760 & $4.90[3.18,5.72]$ & $4.19[3.00,5.26]$ & 0.734 \\
\hline $\begin{array}{l}\text { Platelets }\left(10^{\wedge} 9 / \mathrm{L}\right), \\
\text { median }[\mathrm{IQR}]\end{array}$ & $\begin{array}{l}202.00[157.00, \\
204.00]\end{array}$ & $\begin{array}{l}387.00[272.00, \\
429.00]\end{array}$ & 0.002 & $\begin{array}{l}215.50[169.00 \\
349.50]\end{array}$ & $\begin{array}{l}389.50[272.75, \\
425.25]\end{array}$ & 0.031 \\
\hline $\begin{array}{l}\text { C-reactive protein } \\
(\mathrm{mg} / \mathrm{L}), \text { median } \\
{[\mathrm{IQR}]}\end{array}$ & $61.25[45.35,80.78]$ & $36.10[17.30,61.70]$ & 0.225 & $46.50[28.22,80.78]$ & $36.10[17.30,61.70]$ & 0.473 \\
\hline $\begin{array}{l}\text { LDH (U/L), median } \\
\text { [IQR] }\end{array}$ & $\begin{array}{l}328.50[296.75, \\
358.00]\end{array}$ & $\begin{array}{l}282.50[231.75 \\
363.50]\end{array}$ & 0.447 & $\begin{array}{l}290.00[249.50, \\
328.50]\end{array}$ & $\begin{array}{l}287.00[232.50, \\
368.50]\end{array}$ & 0.963 \\
\hline $\begin{array}{l}\text { D-dimer }(\mathrm{mg} / \mathrm{L}) \text {, } \\
\text { median }[\mathrm{IQR}]\end{array}$ & $1.47[1.18,1.76]$ & $2.40[1.38,4.16]$ & 0.243 & $2.27[2.05,3.12]$ & $2.22[1.33,4.07]$ & 0.979 \\
\hline
\end{tabular}

Significant values are shown in bold

Abbreviations: $B M I$ body mass index, $I C U$ intensive care unit, $L D H$ lactate dehydrogenase, $M E W S$ modified early warning score, $n$ number, qSOFA quick sequential organ failure assessment

${ }^{1}$ Comorbidities include chronic cardiac disease, hypertension, chronic pulmonary disease, asthma, chronic kidney disease, liver disease, chronic neurologic disease, malignancy, chronic hematologic disease, HIV or aids, diabetes, rheumatic disorder, auto-immune disease, and dementia

${ }^{2-5}$ Percentage of missing values: ${ }^{2} 44 \%,{ }^{3} 28 \%,{ }^{4} 14 \%,{ }^{5}$ between 51 and $64 \%$

${ }^{6}$ One of the seven Halm's criteria (the ability to maintain oral intake) was not record

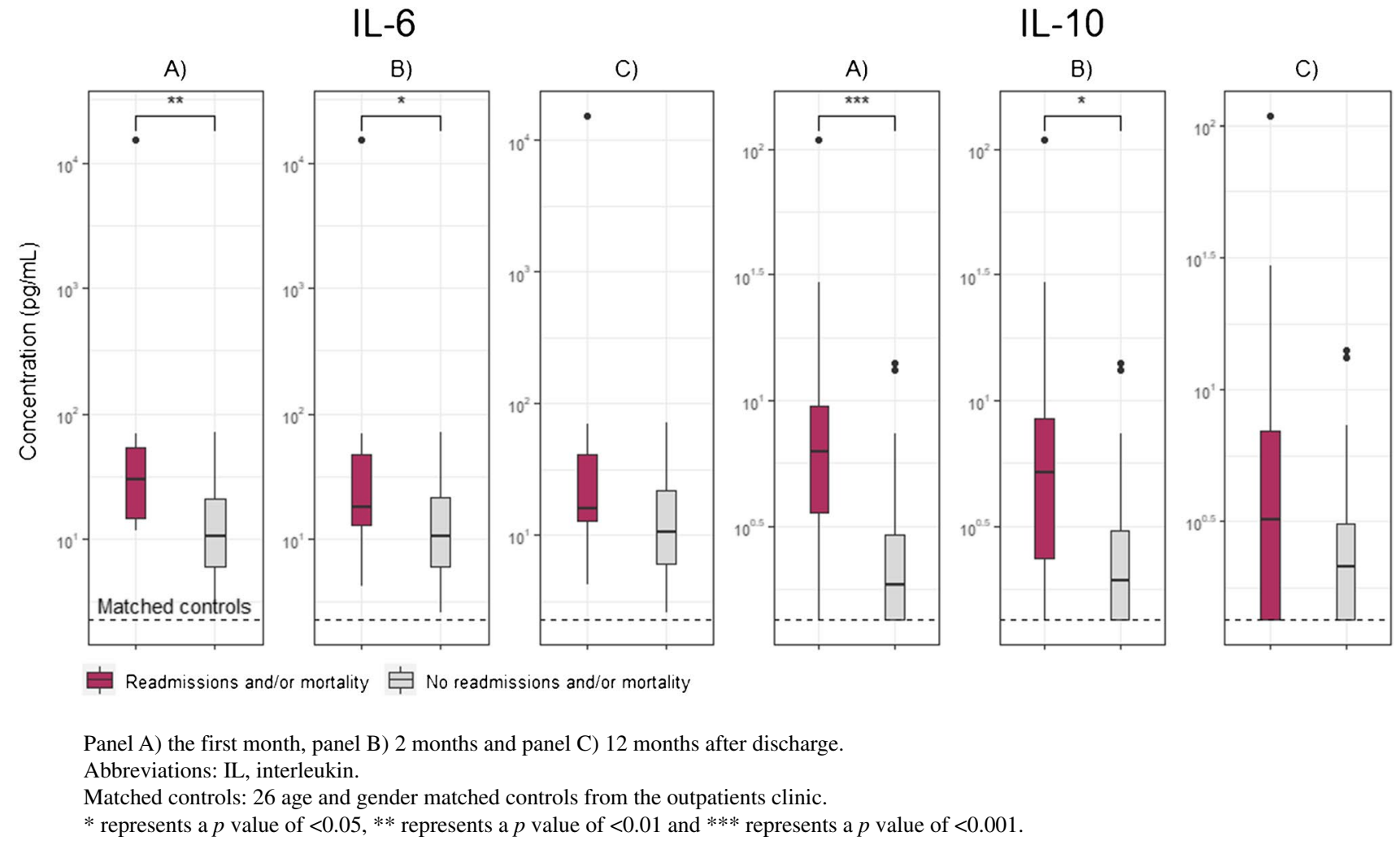

Fig. 1 Concentration interleukin- 6 and interleukin- 10 at hospital discharge, stratified for readmission and/or mortality 
Acknowledgements We would like to thank all medical, paramedical, laboratory, and nursing staff involved in the care of the COVD19 patients for making it possible to build the Amsterdam UMC COVID-19 Biobank in the middle of the COVID-19 outbreak in The Netherlands.

Author contribution All authors contributed to the study conception and design. Material preparation, data collection, and analysis were performed by Marleen A. Slim, Brent Appelman, and Lonneke A. van Vught. The first draft of the manuscript was written by Marleen A. Slim, W. Joost Wiersinga, and Lonneke A. van Vught, and all authors commented on previous versions of the manuscript. All authors read and approved the final manuscript.

Funding This study was funded by the Amsterdam UMC, Amsterdam UMC Corona Research Fund. Lonneke A. van Vught was supported by a VENI grant from ZonMW (grant number 09150161910033).

\section{Declarations}

Conflict of interest The authors declare no competing interests. Collaborators Amsterdam UMC COVID-19 biobank study group See Appendix.

\section{References}

1. Prescott HC, Levy MM (2021) Survival from severe coronavirus disease 2019: is it changing? Crit Care Med 49(2):351-353. https://doi.org/10.1097/CCM.0000000000004753

2. Donnelly JP, Wang XQ, Iwashyna TJ, Prescott HC (2020) Readmission and death after initial hospital discharge among patients with COVID-19 in a large multihospital system. JAMA 325(3):304-306. https://doi.org/10.1001/jama.2020.21465
3. Yende S, D'Angelo G, Kellum JA, Weissfeld L, Fine J, Welch RD et al (2008) Inflammatory markers at hospital discharge predict subsequent mortality after pneumonia and sepsis. Am J Respir Crit Care Med 177(11):1242-1247. https://doi.org/10.1164/rccm. 200712-1777OC

4. de Bruin S, Bos LD, van Roon MA, Tuip-de Boer AM, Schuurman AR, Koel-Simmelinck MJA et al (2021) Clinical features and prognostic factors in Covid-19: a prospective cohort study. EBioMedicine 67:103378. https://doi.org/10.1016/j.ebiom.2021. 103378

5. Halm EA, Fine MJ, Kapoor WN, Singer DE, Marrie TJ, Siu AL (2002) Instability on hospital discharge and the risk of adverse outcomes in patients with pneumonia. Arch Intern Med 162(11):1278-1284. https://doi.org/10.1001/archinte.162.11.1278

6. Wiersinga WJ, Rhodes A, Cheng AC, Peacock SJ, Prescott HC (2020) Pathophysiology, transmission, diagnosis, and treatment of coronavirus disease 2019 (COVID-19): a review. JAMA 324(8):782-793. https://doi.org/10.1001/jama.2020.12839

7. Osuchowski MF, Winkler MS, Skirecki T, Cajander S, ShankarHari M, Lachmann G et al (2021) The COVID-19 puzzle: deciphering pathophysiology and phenotypes of a new disease entity. Lancet Respir Med 9(6):622-642. https://doi.org/10.1016/S22132600(21)00218-6

8. RECOVERY Collaborative Group (2021) Tocilizumab in patients admitted to hospital with COVID-19 (RECOVERY): a randomised, controlled, open-label, platform trial. Lancet 397(10285):1637-1645. https://doi.org/10.1016/S0140-6736(21) 00676-0

9. Guideline Therapeutics and COVID-19: living guideline. World Health Organization. July 2021. https://apps.who.int/iris/bitstram/ ahndle/10655/432368/WHO-2019-nCoV-therapeutics-2021.2eng.pfd (accessed 17 Aug 2021)

Publisher's note Springer Nature remains neutral with regard to jurisdictional claims in published maps and institutional affiliations. 